\title{
Amino Acid Biosynthesis
}

National Cancer Institute

\section{Source}

National Cancer Institute. Amino Acid Biosynthesis. NCI Thesaurus. Code C30017.

Amino Acid Biosynthesis consists of cellular enzymatic reactions that produce amino acids, org anic compounds containing an amino and a carboxyl group and often used in protein synthesis by the formation of peptide bonds during ribosomal translation of mRNA. Several important amino acids, such as the neurotransmitter g-aminobutyric acid, have other functions than protein synthesis. 